\title{
Measurements of jet fragmentation and jet substructure with ALICE
}

\section{Markus Fasel for the ALICE Collaboration*}

Oak Ridge National Laboratory, Oak Ridge, Tennessee, United States

E-mail: markus.faselecern.ch

\begin{abstract}
We discuss the latest results from jet fragmentation and jet substructure measurements performed with the ALICE experiment in proton-proton and heavy-ion collisions in a wide range of jet transverse momentum. The jet production cross sections and cross section ratios for different jet resolution parameters will be shown in a wide range of $p_{\mathrm{T}}$. Results will be compared to next-to-leading order pQCD calculations.
\end{abstract}

7th Annual Conference on Large Hadron Collider Physics - LHCP2019

20-25 May, 2019

Puebla, Mexico

${ }^{*}$ Speaker. 


\section{Introduction}

Jet substructure measurements at low transverse momentum $p_{\mathrm{T}}$ allow to constrain various perturbative and non-perturbative effects contributing to jet production, such as hadronization, perturbative radiation and the underlying event. For example the measurement of the jet cross section for various jet resolution parameters $R$ and the ratios of cross sections, in particular for $p_{\mathrm{T}}<100 \mathrm{GeV} / c$ show different sensitivity to the various effects [1]. In addition, the large datasets obtained with LHC Run2 allow for more differential studies. This is used in the measurement of the groomed momentum fraction $z_{\mathrm{g}}$ using the SoftDrop algorithm [2,3], defined as

$$
z_{\mathrm{g}}=\frac{\min \left(p_{\mathrm{T}, 1}, p_{\mathrm{T}, 2}\right)}{p_{\mathrm{T}, 1}+p_{\mathrm{T}, 2}}>z_{\text {cut }} \theta^{\beta}
$$

with $p_{\mathrm{T}, 1}$ and $p_{\mathrm{T}, 2}$ being the $p_{\mathrm{T}}$ of the hardest and second hardest subjet. The level of grooming is controlled by the parameters $z_{c u t}$ and $\beta$. The observable is selected as it is closely related to the QCD splitting function at leading order [1]. No $p_{\mathrm{T}}$-dependence is expected. The difference between the parton and the reconstructed jet momentum is expected to increase as $\log (R)$ for perturbative radiation, to decrease as $1 / R$ due to hadronization effects and to increase as $\sim R^{2}$ due to the contribution from the underlying event [1]. In heavy-ion collisions the groomed momentum fraction is sensitive to effects from the hot and dense medium.

Jets are reconstructed using the anti- $k_{\mathrm{T}}$ algorithm [4] from the FastJet package [5] employing the E scheme recombination. Clustering is done for track-based jets using tracks reconstructed in the ALICE tracking detectors, requiring a minimum constituent $p_{\mathrm{T}}$ of $150 \mathrm{MeV} / c$. For fully reconstructed jets clusters in the electromagnetic calorimeter with energy larger than $300 \mathrm{MeV}$ are included in the jet reconstruction.

The data used for the measurement in pp collisions at $\sqrt{s}=13 \mathrm{TeV}$ was collected in 2016 and 2017, consisting of a min. bias dataset with $L_{\mathrm{int}}=11.5 \mathrm{nb}^{-1}$ and a jet-triggered dataset based on energy deposition in the electromagnetic calorimeter with $L_{\text {int }}=4 \mathrm{pb}^{-1}$. For the measurements in heavy-ion collisions the data was collected at $\sqrt{{ }^{s} \mathrm{NN}}=2.76 \mathrm{TeV}$ in 2011 and consists of $16 \mathrm{M}$ central collisions.

In heavy-ion collisions jet reconstruction is affected by the particles from the underlying event. Therefore observables with low sensitivity to the background, like jet-hadron correlations [6] are preferential. For substructure measurements the effect of the uncorrelated background can be handled in the detector response. For this PYTHIA events [7, 8] are embedded into heavy-ion events, and the effect of the background is estimated and subtracted using area based [9] or constituent based [10] subtraction methods.

\section{Measurement of the jet production in pp collisions at $\sqrt{s}=13 \mathrm{TeV}$}

The $p_{\mathrm{T}}$-differential cross section of jet production is measured for different jet radii between $R=0.2$ and $R=0.6$ for $20 \mathrm{GeV} / c<p_{\mathrm{T}}<320 \mathrm{GeV} / c$ (Fig. 1 (left)). The comparison to POWHEG+PYTHIA6 [11, 12, 13] is shown in Fig. 2 for $R=0.2$ (left) and $R=0.4$ (right). POWHEG calculations use $\mu_{R}=\mu_{F}=1$. Calculations are in good agreement with the measured production cross sections. The ratios of the differential jet production cross section for jets with 

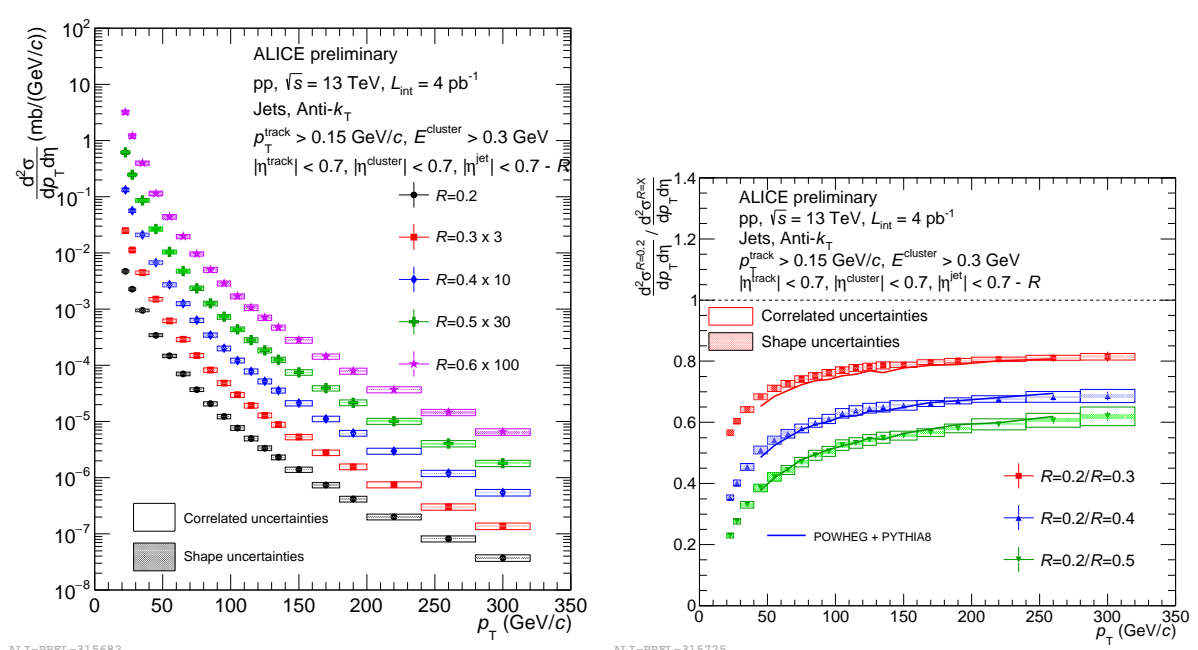

Figure 1: Left: $p_{\mathrm{T}}$-differential jet production cross section in pp collisions at $\sqrt{\mathrm{s}}=13 \mathrm{TeV}$ for different $R$. Right: Ratios of jet production cross sections for jets with $R=0.2$ to the cross section for various $R$. Lines indicate calculations using POWHEG+PYTHIA6.
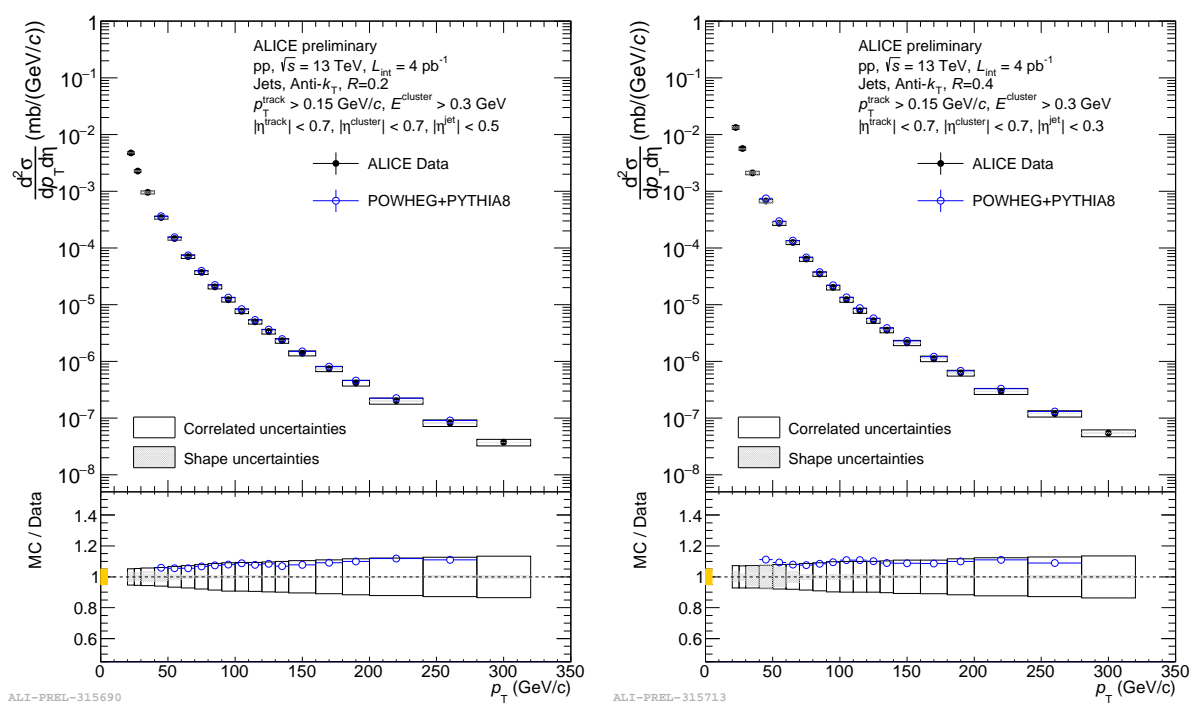

Figure 2: $p_{\mathrm{T}}$-differential jet production cross section for jets with $R=0.2$ (left) and $R=0.4$ (right) compared to POWHEG+PYTHIA6 $\left(\mu_{R}=\mu_{F}=1\right)$.

$R=0.2$ to the cross section for various $R$ is shown in Fig. 1 (right) for $R=0.3$ to $R=0.6$ in the same $p_{\mathrm{T}}$ range. As many of the systematic uncertainties cancel in the ratios they can be measured with a higher precision. The cross section ratios are well-described by POWHEG+PYTHIA6. A good agreement with POWHEG+PYTHIA6 has also been found in cross section measurements of track-based jets and fully reconstructed jets at lower centre-of-mass energies [14, 15, 16]. 


\section{Measurement of the groomed momentum fraction $z_{g}$ in pp and heavy-ion collisions}

In pp collisions at $\sqrt{s}=13 \mathrm{TeV}$ the groomed momentum fraction $z \mathrm{~g}$ has been measured for jets with $30 \mathrm{GeV} / c<p_{\mathrm{T}}<200 \mathrm{GeV} / c$ for various ranges in $p_{\mathrm{T}}$. Jets were reclustered with the Cambridge/Aachen algorithm. The SoftDrop parameters $\beta=0$ and $z \mathrm{~g}=0.1$ were used. No underlying event subtraction has been applied as the grooming already removes soft components from the underlying event. The distributions are unfolded back to particle level using a 2-dimensional unfolding based on the Bayesian method [17]. The response matrices have been obtained using a full detector simulation based on GEANT3 [18] of pp events at the same centre-of-mass energy generated with PYTHIA8 [8].
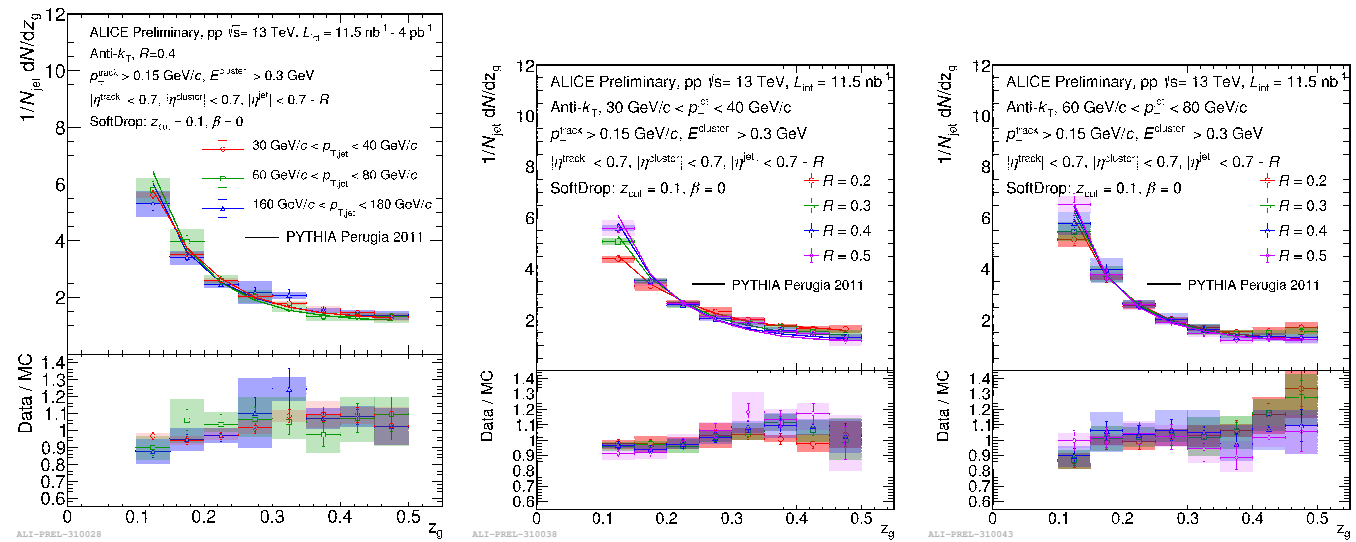

Figure 3: Left: $z_{\mathrm{g}}$-differential per-jet yield for jets with $R=0.4$ for different bins in $p_{\mathrm{T}}$ (left), jets with different $R$ for $30 \mathrm{GeV} / c<p_{\mathrm{T}}<40 \mathrm{GeV} / c$ (middle), and jets with different $R$ for $60 \mathrm{GeV} / c<p_{\mathrm{T}}<$ $80 \mathrm{GeV} / c$ (right). Lines indicate calculations with the PYTHIA event generator using the Perugia 0 tune [19].

Fig. 3 (left) shows the $z_{g}$ - differential jet yield for jets with $\mathrm{R}=0.4$ for $30 \mathrm{GeV} / c<p_{\mathrm{T}}<$ $40 \mathrm{GeV} / c, 60 \mathrm{GeV} / c<p_{\mathrm{T}}<80 \mathrm{GeV} / c$, and $160 \mathrm{GeV} / c<p_{\mathrm{T}}<180 \mathrm{GeV} / c$. Results agree within the uncertainties, indicating no $p_{\mathrm{T}}$-dependence of the $z \mathrm{~g}$ for jets with large resolution parameter. In the middle and right panel the $\mathrm{zg}$-differential yield of jets is plotted for different $R$ from $R=0.2$ to $R=0.4$ for jets with $30 \mathrm{GeV} / c<p_{\mathrm{T}}<40 \mathrm{GeV} / c$ (middle) and $60 \mathrm{GeV} / c<p_{\mathrm{T}}<80 \mathrm{GeV} / c$ (right). For jets with lower $p_{\mathrm{T}}$ a difference in the $z \mathrm{~g}$-differential yield for different $R$ is observed, tending towards more symmetric splitting for jets with smaller $R$. For jets with higher $p_{\mathrm{T}}$ no dependence of the $\mathrm{zg}$ on $R$ is observed. The trend is well reproduced by PYTHIA6 using the Perugia 0 tune.

In $\mathrm{Pb}$ - $\mathrm{Pb}$ collisions the $z \mathrm{~g}$-differential yield of track based jets was measured at $\sqrt{{ }^{\mathrm{NN}}}=2.76$ $\mathrm{TeV}$ for jets with $R=0.4$ and $80 \mathrm{GeV} / c<p_{\mathrm{T}}<120 \mathrm{GeV} / c$ [20]. Fig. 4 shows the $z \mathrm{~g}$ - differential yields for strongly collimated jets with $\Delta R<0.1$, where $\Delta R$ is the distance between the subjets, and jets with $\Delta \mathrm{R}>0.2$. For the comparison to the vacuum PYTHIA events were embedded into $\mathrm{PbPb}$ events in order to account for effects from the underlying background. While no modification can be observed for very collimated subjets, for jets with larger $\Delta R$ a suppression of jets with symmetric splittings with an enhancement of untagged jets can be observed. 

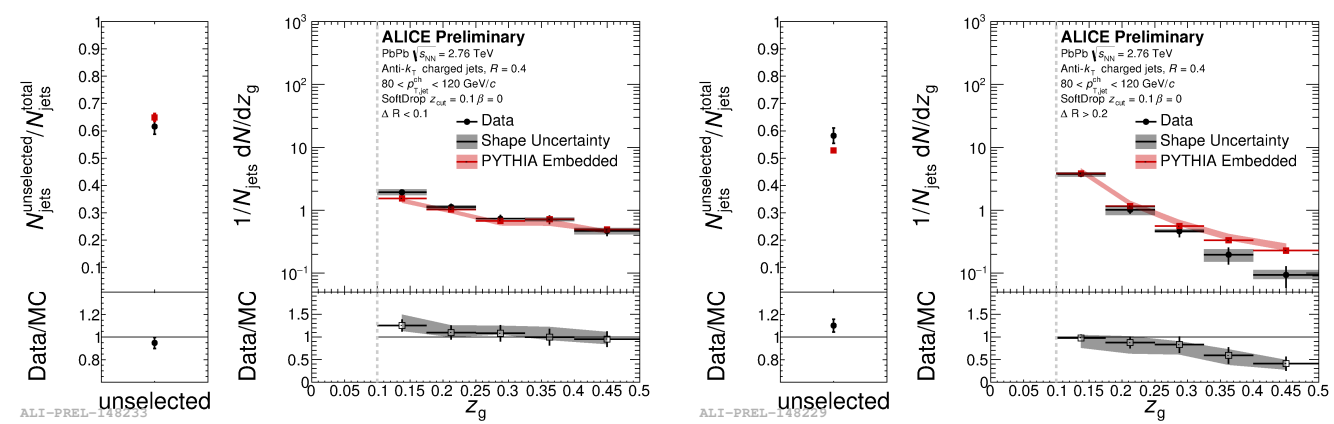

Figure 4: $z \mathrm{~g}$-differential yield of track-based jets with $80 \mathrm{GeV} / c<p_{\mathrm{T}}<120 \mathrm{GeV} / c$ and $R=0.4$. [20] Left: jets with $\Delta R<0.1$. Right: jets with $\Delta \mathrm{R}>0.2$. Red bands indicating the distributions from PYTHIA events embedded into heavy-ion collisions.

\section{Outlook}

The study of jet substructure can be generalized by varying the grooming conditions or looking at all splittings and constructing the Lund plane [21]. The Lund plane is obtained directly from the QCD kernel. Recent studies [22] show that different regions in the Lund plane are sensitive to different effects, among them medium-induced radiation and coherent radiation. A precise measurement of the Lund plane in heavy-ion collisions will allow to provide further constraints on the different effects contributing to jet modification in the hot and dense medium.

\section{References}

[1] M. Dasgupta, F. A. Dreyer, G. P. Salam and G. Soyez, Inclusive jet spectrum for small-radius jets, JHEP 06 (2016) 057 [1602.01110].

[2] A. J. Larkoski, S. Marzani, G. Soyez and J. Thaler, Soft Drop, JHEP 05 (2014) 146 [1 402 . 2657].

[3] J. M. Butterworth, A. R. Davison, M. Rubin and G. P. Salam, Jet substructure as a new Higgs search channel at the LHC, Phys. Rev. Lett. 100 (2008) 242001 [0802 . 2470].

[4] M. Cacciari, G. P. Salam and G. Soyez, The anti- $k_{t}$ jet clustering algorithm, JHEP 04 (2008) 063 [0802.1189].

[5] M. Cacciari, G. P. Salam and G. Soyez, FastJet User Manual, Eur. Phys. J. C72 (2012) 1896 [1111.6097].

[6] ALICE collaboration, J. Adam et al., Measurement of jet quenching with semi-inclusive hadron-jet distributions in central Pb-Pb collisions at $\sqrt{s_{\mathrm{NN}}}=2.76$ TeV, JHEP 09 (2015) 170 [1506.03984].

[7] T. Sjostrand, S. Mrenna and P. Z. Skands, PYTHIA 6.4 Physics and Manual, JHEP 05 (2006) 026 [hep-ph/0603175].

[8] T. SjÃústrand, S. Ask, J. R. Christiansen, R. Corke, N. Desai, P. Ilten et al., An Introduction to PYTHIA 8.2, Comput. Phys. Commun. 191 (2015) 159 [1410.3012].

[9] G. Soyez, G. P. Salam, J. Kim, S. Dutta and M. Cacciari, Pileup subtraction for jet shapes, Phys. Rev. Lett. 110 (2013) 162001 [1211.2811]. 
[10] P. Berta, M. Spousta, D. W. Miller and R. Leitner, Particle-level pileup subtraction for jets and jet shapes, JHEP 06 (2014) 092 [1403.3108].

[11] S. Frixione, P. Nason and C. Oleari, Matching NLO QCD computations with Parton Shower simulations: the POWHEG method, JHEP 11 (2007) 070 [0 709.2092 ].

[12] S. Alioli, P. Nason, C. Oleari and E. Re, A general framework for implementing NLO calculations in shower Monte Carlo programs: the POWHEG BOX, JHEP 06 (2010) 043 [1 002 . 2 581].

[13] S. Alioli, K. Hamilton, P. Nason, C. Oleari and E. Re, Jet pair production in POWHEG, JHEP 04 (2011) 081 [1012.3380].

[14] ALICE collaboration, J. Mulligan, Inclusive jet measurements in pp and Pb-Pb collisions with ALICE, PoS HardProbes2018 (2019) 080 [1812 . 07681 ].

[15] ALICE collaboration, S. Acharya et al., Measurement of charged jet cross section in pp collisions at $\sqrt{s}=5.02 \mathrm{TeV}, 1905.02536$.

[16] ALICE collaboration, S. Acharya et al., Charged jet cross section and fragmentation in proton-proton collisions at $\sqrt{s}=7$ TeV, Phys. Rev. D99 (2019) 012016 [1809. 03232].

[17] G. D'Agostini, A multidimensional unfolding method based on bayes' theorem, Nuclear Instruments and Methods in Physics Research Section A: Accelerators, Spectrometers, Detectors and Associated Equipment 362 (1995) 487 .

[18] R. Brun, F. Bruyant, M. Maire, A. McPherson and P. Zanarini, GEANT3, .

[19] P. Z. Skands, Tuning Monte Carlo Generators: The Perugia Tunes, Phys. Rev. D82 (2010) 074018 [1005.3457].

[20] ALICE collaboration, S. Acharya et al., Exploration of jet substructure using iterative declustering in pp and Pb-Pb collisions at LHC energies, 1905.02512.

[21] B. Andersson, G. Gustafson, L. Lonnblad and U. Pettersson, Coherence Effects in Deep Inelastic Scattering, Z. Phys. C43 (1989) 625.

[22] H. A. Andrews et al., Novel tools and observables for jet physics in heavy-ion collisions, 1808.03689. 